\title{
Encephalitis-Associated Pandemic A (H1N1) 2009 in a Kuwaiti Girl
}

\author{
Fawaz Al-Baghli Wafaa Al-Ateeqi \\ Department of Pediatrics, Al-Amiri Hospital, Kuwait City, Kuwait
}

\section{Key Words}

Encephalitis · Influenza $\cdot$ Pandemic A (H1N1)

\begin{abstract}
Objective: To report an 11-year-old girl with encephalitis-associated pandemic influenza A (H1N1) 2009 virus infection. Clinical Presentation and Intervention: An 11-year-old girl presented with a 6-day history of influenza-like illness followed by an altered mental status for 1 day. She tested positive for pandemic influenza A (H1N1) 2009 virus by PCR of her nasal swab. Her CSF analysis was completely normal, including PCR for pandemic influenza $A$ virus. Her brain MRI and EEG showed evidence suggestive of encephalitis. She was treated with oral Oseltamivir with good recovery. Conclusion: This case report shows that neurological complications can occur after respiratory tract infection with pandemic influenza A (H1N1) 2009 virus. Copyright $\odot 2011$ S. Karger AG, Basel
\end{abstract}

\section{Introduction}

Pandemic influenza A (H1N1) 2009 virus was documented for the first time in humans in North America in April 2009. Subsequently, it spread rapidly to the rest of the world and was declared to be a pandemic infection [1].
A variety of clinical manifestations have been observed, the vast majority are respiratory symptoms. However, other systems such as the central nervous system were found to be affected [2]. We report a case of an 11-year-old girl who had encephalitis associated with pandemic influenza A (H1N1) 2009 virus infection.

\section{Case Report}

An 11-year-old girl, previously healthy, presented to the pediatric emergency in Al-Amiri hospital, Kuwait, with a history of fever for 6 days and drowsiness of 1 day's duration. At the beginning of her illness, she was diagnosed with upper respiratory tract infection and received oral cefuroxime with no improvement. There was no history of convulsions, headache, or photophobia. She had good school performance and normal development. On the fifth day of her illness, pandemic influenza A (H1N1) 2009 was suspected and she tested positive by reverse-transcription PCR on a nasal swab. Cefuroxime was stopped and oral oseltamivir was started. One day prior to admission she became persistently sleepy, lethargic and was unable to attend her needs. She continued to be drowsy and required admission to hospital. At the same time, her sister, who had a mild upper respiratory tract infection, was also found to be positive for pandemic influenza A (H1N1) 2009, but she did not require hospital admission.

On physical examination, her weight was $29 \mathrm{~kg}$ (10th percentile), height $145 \mathrm{~cm}$ (50th percentile). She was drowsy and lethargic during the examination. The Glasgow Coma Scale was 11/15 (eyes: opening spontaneously -4 ; motor: withdrawal to pain -4 ;

\section{KARGER}

Fax +4161306 1234

E-Mail karger@karger.ch

www.karger.com (c) 2011 S. Karger AG, Basel

1011-7571/11/0202-0191\$38.00/0

Accessible online at:

www.karger.com/mpp
Dr. Fawaz Al-Baghli

P.O. Box 39089. Al-Nuzha

73051 Kuwait City (Kuwait)

Tel. +965 9907 7337, Fax +965 24926097

E-Mail fwz.albag@gmail.com 

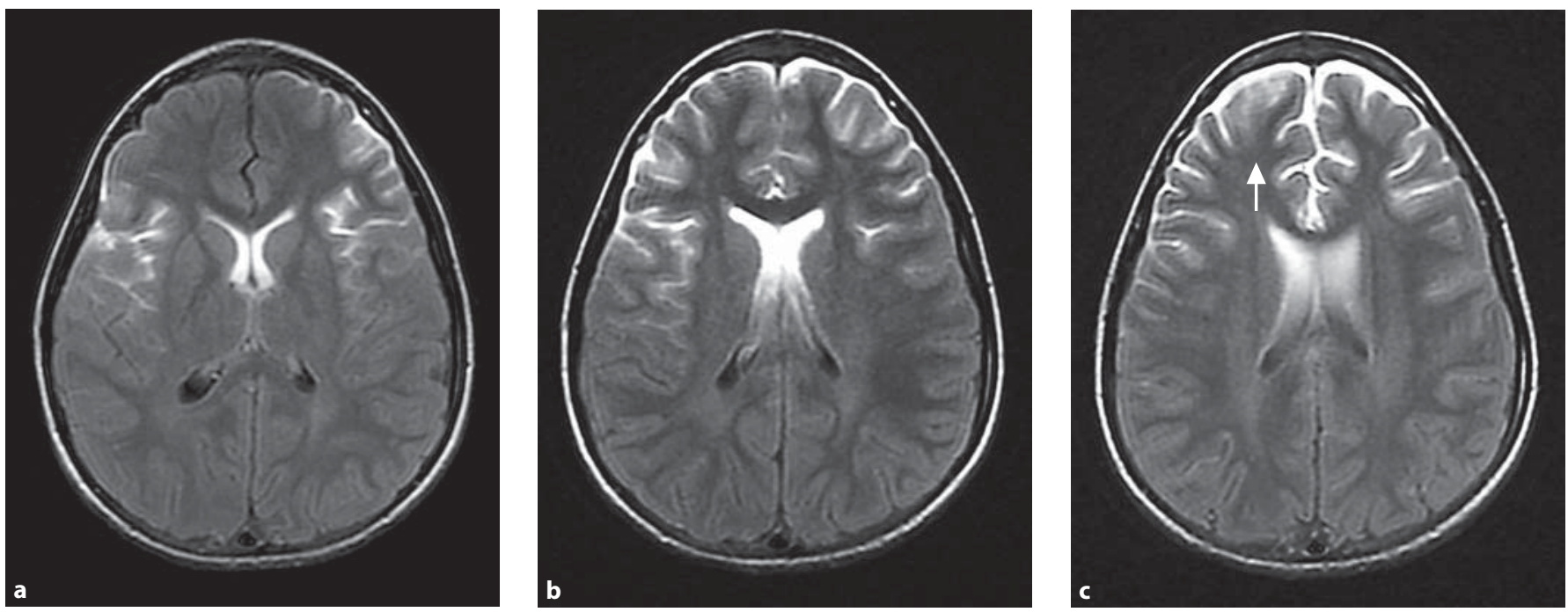

Fig. 1. a-e Sequentially adjacent, axial sections of brain MRI on FLAIR sequence. The arrow in c points to an area of focal bright signal intensity and blur of cortical ribbon on the right frontal lobe.
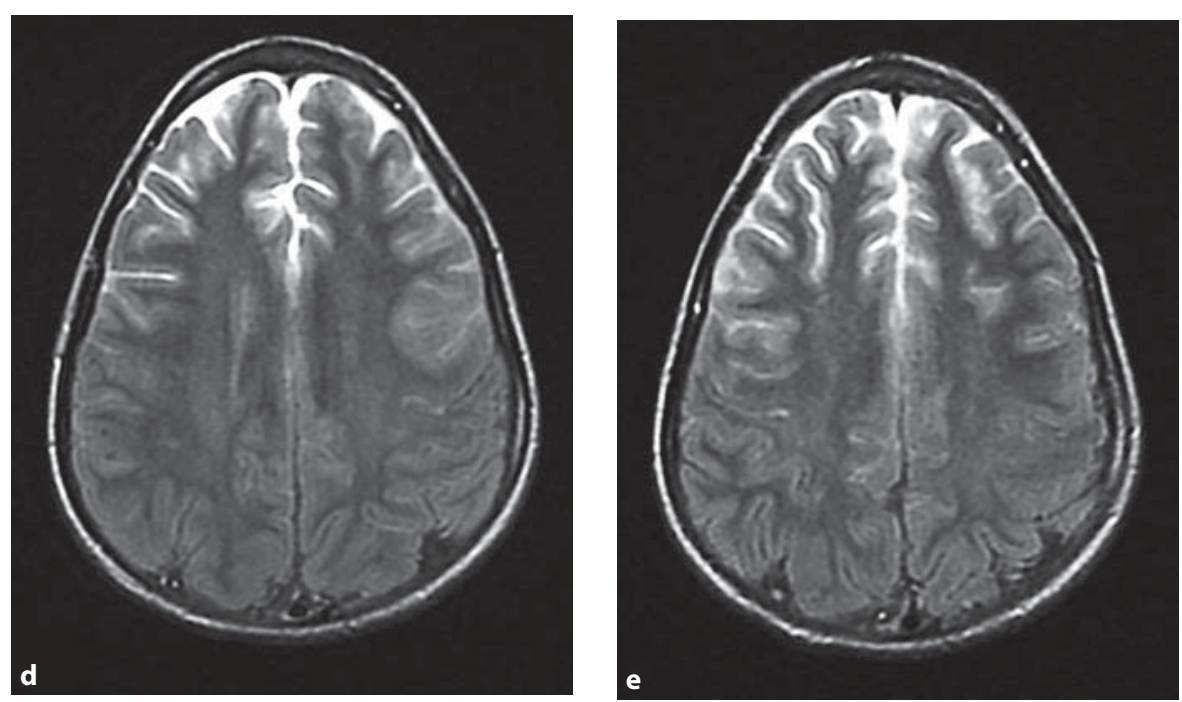

verbal: incoherent words - 3). Her temperature was $38^{\circ} \mathrm{C}$, heart rate was 116 beats/min; the $\mathrm{O}_{2}$ saturation was $98 \%$ in room air and blood pressure was 100/70 $\mathrm{mm} \mathrm{Hg}$. There was no skin rash. Central nervous system examination showed normal tone, power of $5 / 5$ in 4 limbs, normal reflexes, and intact cranial nerves. There were no signs of meningeal irritation, pupils were equal and reactive, and there was no papiloedema. Examination of other organs was unremarkable. Soon after admission she was transferred to the pediatric ICU. Meningoencephalitis was suspected and full septic workup was performed, including complete blood count, C-reactive protein, blood and urine cultures, CSF for biochemistry and culture, virology tests by PCR on blood, nasal swab, and CSF. She was started empirically on i.v. acyclovir $(60 \mathrm{mg} / \mathrm{kg} / \mathrm{day})$, i.v. cefotaxime ( $200 \mathrm{mg} / \mathrm{kg} /$ day), and oral oseltamivir ( $90 \mathrm{mg} /$ day) was continued. Laboratory investigations were as follows. Complete blood count: WBC $4.2 \times 10^{9} / 1$ (23\% neutrophils, $70 \%$ lymphocytes) with an absolute neutrophil count of $1.0 \times 10^{9} / 1$, hemo- globin $139 \mathrm{~g} / \mathrm{l}$, platelets $162 \times 10^{9} / 1$. Renal and liver function tests were normal. C-reactive protein: negative. Coagulation profile: normal. Nasal swab specimen for pandemic influenza A (H1N1) test (by reverse-transcription PCR) was repeated and it was positive. Nasal swab was tested for other viruses (adenovirus, influenza b virus, para-influenza virus, rhinovirus, corona, and RSV virus) and was negative. A CSF analysis was taken on the seventh day of the illness: WBC $<4 \mathrm{~mm}^{3}, \mathrm{RBC}<4 \mathrm{~mm}^{3}$, protein $549 \mathrm{mg} / \mathrm{l}$, glucose $2.8 \mathrm{mmol} / \mathrm{l}$, lactate dehydrogenase $18 \mathrm{U} / \mathrm{l}$, lactate 1.35 $\mathrm{mmol} / \mathrm{l}$. Direct smear revealed no micro-organisms. Tests for bacterial antigens were negative. Culture was negative after $48 \mathrm{~h}$. CSF was negative for pandemic influenza A (H1N1) virus, herpes simplex virus, human herpes virus 6, and enterovirus by PCR; Varicella-Zoster was not tested.

MRI of her head with contrast showed a focal area of bright signal intensity with blurring of cortical ribbon seen on T2W1 and FLAIR sequence. This is seen as low signal intensity on T1W1 


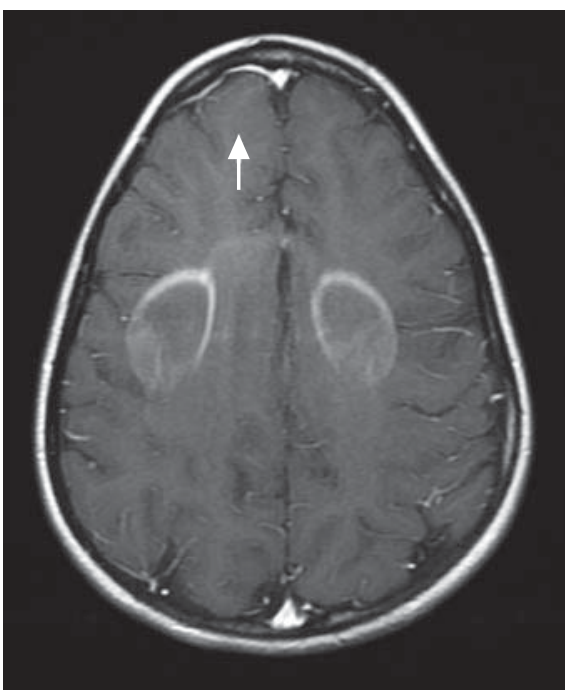

Fig. 2. Post-contrast axial brain MRI. The arrow points to an area of focal linear meningeal enhancement.

(fig. 1). Post-contrast study revealed only focal linear meningeal enhancement in the right frontal region. There was no evidence of parenchymal enhancement (fig. 2). These features were consistent with the diagnosis of meningoencephalitis. EEG showed a generalized slowing suggestive of an encephalopathic process.

With this clinical picture, results of nasal swab and MRI finding, encephalitis was suspected, most likely due to pandemic influenza A (H1N1) 2009 influenza infection. The patient received oral oseltamivir for 5 days, i.v. cefotaxime, and i.v. acyclovir were discontinued once the cultures and virology test became negative. While in the pediatric ICU, she had acutely raised creatinine levels $(122 \mathrm{umol} / \mathrm{l})$. This was thought to be secondary to i.v. medication (acyclovir) or i.v. contrast. It was managed by i.v. fluid, and the dose of acyclovir was reduced. The rest of her renal function tests were normal.

She stayed in the pediatric ICU for 4 days and was then transferred to the ward. In the ward, she was conscious with a Glasgow Coma Scale score of 15/15. She looked generally weak, not interested in talking and avoiding eye contact. Her speech was monotonic. She had a mild tremor and incoordination of her limbs. She gradually improved and on discharge returned to her normal behavior and activity. Her central nervous system examination was normal.

\section{Discussion}

Neurological complications associated with seasonal influenza virus have been reported for more than 100 years. Historical records reveal that in 1918, more than 40 million people died due to an influenza pandemic, which was rapidly followed by an epidemic of encephalitis le- thargica in 1919 [3]. The capability of seasonal influenza virus to cause neurological complications is well documented in the literature. These complications include influenza-associated encephalitis/encephalopathy, myelitis, seizures, Reye syndrome and acute necrotizing encephalitis [4].

Influenza virus infection was associated with $5 \%$ of cases of acute childhood encephalitis/encephalopathy [5]. The majority of these children were $<5$ years of age. The prevalence of neuroimaging abnormalities was higher in children $<2$ years of age, suggesting that younger children are more predisposed to the neurologic complications of influenza. In addition, acute rather than a postinfectious process was suggested by the briefness of the respiratory prodrome in most cases [5]. The epidemiology of influenza-associated encephalopathy has been described extensively in Japan where the incidence has appeared to be higher than in other countries [4]. A national survey of influenza-associated encephalopathy was carried out in Japan between 1998 and 2002. This survey found that the majority of patients had relatively minor neurological symptoms. However, a small but significant number experienced serious complications, resulting in neurologic sequelae or even death [6]. This highlights the seriousness of neurologic complications of the influenza virus. The pathogenesis of influenza-associated encephalitis/encephalopathy is unclear, and whether the influenza virus invades the brain parenchyma is still a controversial issue. Viral RNA was frequently detected in the CSF by reverse-transcription PCR [4]. However, recent reports have indicated that viral RNA is not detected in the CSF of most patients with influenza-associated encephalitis/encephalopathy [4]. Influenza A virus strains with certain hemagglutinin $(\mathrm{H})$ and neuraminidase $(\mathrm{N})$ profiles are more often associated with CNS complications; in particular, influenza A (H3N2) virus infections have been found in the majority of encephalopathy cases reported in Japan and Europe [6]. Avian influenza A $\mathrm{H} 5 \mathrm{~N} 1$ virus has been shown to target the nervous system in post-mortem studies. Its viral genomic sequences and antigens were detected in neurons of the brain as well as other organs in fatal cases of avian A H5N1 [7].

To date, there are a number of reports of neurologic complications associated with the pandemic influenza A (H1N1) 2009 virus infection. The first report describing patients with neurologic complications associated with pandemic influenza A (H1N1) 2009 virus infection was from Dallas, Tex., USA, in May 2009 [2]. In this report, a patient with acute neurologic complications associated with pandemic influenza A (H1N1) 2009 virus infection 
was defined as having laboratory-confirmed pandemic influenza A (H1N1) 2009 virus infection of the respiratory tract associated with seizures, encephalopathy, or encephalitis within 5 days of influenza-like illness symptoms, without evidence of an alternative etiology. Encephalopathy was defined as altered mental status lasting for $24 \mathrm{~h}$ and encephalitis was defined as encephalopathy plus 2 or more of the following: fever of $\geq 38.0^{\circ} \mathrm{C}$, focal neurologic signs, CSF pleocytosis, EEG indicative of encephalitis or abnormal neuroimaging indicative of infection or inflammation. In this report, 4 patients had acute neurologic complications associated with pandemic influenza A (H1N1) 2009 virus. The patients were between 7 and 17 years of age and all had confirmed pandemic influenza A (H1N1) 2009 in nasopharyngeal specimens but not in CSF. Three of 4 patients had abnormal EEG. All patients had normal brain imaging (CT/MRI) and all were treated with anti-influenza medications (oseltamivir \pm rimantadine) and had good recovery with no neurologic sequelae on discharge. One patient was discharged on anti-epileptic medication until follow-up [2].

Two patients with severe neurological complications associated with pandemic influenza A (H1N1) 2009 virus infection were reported by Webster et al. [8]. Both patients were younger than 6 years old. The first had hemi-paresis and seizures and the second ascending paralysis and weakness, progressing to complete paralysis and coma within $24 \mathrm{~h}$. Both required intubation and mechanical ventilation and had positive nasal swabs for pandemic influenza A (H1N1) 2009 virus, but a negative CSF PCR for H1N1 and completely normal CSF glucose, protein, and cell count. Other infectious agents were ruled out. Both cases received treatment with oral oseltamivir and systemic steroids. The second case received i.v. immunoglobulin as well. The first had a full recovery within a week, whereas the second had mild left arm weakness a month later. Sánchez-Torrent et al. [9] reported a case of a 3-month-old infant with upper respiratory tract infection followed by repeated seizures. Pandemic influenza A (H1N1) 2009 virus was isolated in both the nasopharyngeal aspirate and CSF fluid. This was the only case we found in the literature with a positive CSF for pandemic influenza A (H1N1) 2009 virus. The patient was treated with oral oseltamivir and had no neurologic sequelae. Another case of an adolescent with neuropsychiatric symptoms associated with pandemic influenza A (H1N1) 2009 virus was reported by GermanDiaz et al. [10].

Our patient had symptoms of influenza-like illness for 5 days followed by altered mental status for $24 \mathrm{~h}$. She had abnormal neuroimaging and EEG, and a completely normal CSF analysis. Pandemic influenza A (H1N1) 2009 virus was isolated in her nasal swab. She was treated with oral oseltamivir and made a full recovery. This is another case of this rare complication of the pandemic influenza A (H1N1) 2009 virus.

Neuroimaging results in influenza-associated encephalopathy might be normal, but in severe cases abnormalities can include diffuse cerebral edema and bilateral thalamic lesions. EEG might show diffuse abnormalities.

Our case, as well as previously reported cases, should alert clinicians to the potential for neurologic manifestation with the influenza virus. Therefore, clinicians should send respiratory specimens for appropriate analysis. Antiviral treatment should be initiated as soon as possible for any hospitalized patient with neurologic symptoms and suspected influenza or pandemic influenza $\mathrm{A}(\mathrm{H} 1 \mathrm{~N} 1)$ 2009 virus infection. Antiviral medications have been shown to decrease the risk of complications from influenza. However, their effectiveness in preventing influenza-associated encephalopathy sequelae is unknown.

\section{Conclusions}

For children who have influenza-like illness accompanied by unexplained seizures or mental status changes, clinicians should consider acute seasonal influenza or pandemic influenza A (H1N1) 2009 virus infection in the differential diagnosis. Respiratory specimens should be send for appropriate diagnostic tests, and empirical antiviral treatment should be initiated promptly. Febrile seizures should also be considered in the differential diagnosis of young children. Furthermore, until the diagnosis of herpes encephalitis is ruled out, i.v. acyclovir should be included in the treatment plan.

\section{Acknowledgment}

The authors would like to thank Dr. Lamya Al-Rabiah, Radiology Department, Al-Sabah Hospital, for providing us with the patient's MRI images and their interpretation, and Dr. Wedad AlNaqeeb, Virology Department, Kuwait University, for testing the CSF for viruses including Pandemic A (H1N1) 2009 virus. 


\section{References}

1 Pandemic (H1N1) 2009. WHO Global Alert and Response website. www.who.int/csr/disease/swineflu/en/ (accessed October 4, 2010).

2 Evans AS, Agadi MD, Siegel MD: Neurologic complications associated with novel influenza A (H1N1) virus infection in children. MMWR Morb Mortal Wly Rep 2009;58: 773-778.

-3 Tamaki R, Kamigaki T, Oshitani H: Encephalitis and encephalopathy associated with pandemic flu. Brain Nerve 2009;61: 153-160.

4 Morishima T, Togashi T, Yokota S, Okuno Y, Miyazaki C, Tashiro M, Okabe N; Collaborative Study Group on Influenza-Associated Encephalopathy in Japan: Encephalitis and encephalopathy associated with an influenza epidemic in Japan. Clin Infect Dis 2002; 35:512-517.
5 Amin R, Ford-Jones E, Richardson SE, MacGregor D, Tellier R, Heurter H, Fearon M, Bitnun A: Acute childhood encephalitis and encephalopathy associated with influenza: a prospective 11-year review. Pediatr Infect Dis J 2008;27:390-395.

6 Maricich SM, Neul JL, Lotze TE, Cazacu AC, Uyeki TM, Demmler GJ, Clark GD: Neurologic complications associated with influenza A in children during the 2003-2004 influenza season in Houston, Texas. Pediatrics 2004;114:e626-e633.

7 Gu J, Xie Z, Gao Z, Liu J, Korteweg C, Ye J, Lau LT, Lu J, Gao Z, Zhang B, McNutt MA, Lu M, Anderson VM, Gong E, Yu AC, Lipkin WI: H5N1 infection of the respiratory tract and beyond: a molecular pathology study. Lancet 2007;370:1137-1145.
-8 Webster RI, Hazelton B, Suleiman J, Macartney K, Kesson A, Dale RC: Severe encephalopathy with swine origin influenza A H1N1 in childhood: case reports. Neurology 2010; 74:1077-1078.

$\checkmark 9$ Sánchez-Torrent L, Triviño-Rodriguez M, Suero-Toledano P, Claret-Teruel G, MuñozAlmagro C, Martínez-Sánchez L, JordánGarcía I, Garcia-Garcia JJ: Novel influenza A (H1N1) encephalitis in a 3-month-old infant. Infection 2010;38:227-229.

10 German-Diaz M, Pavo-Garcia R, Diaz-Diaz J, Giangaspro-Corradi E, Negreira-Cepeda $S$ : Adolescent with neuropsychiatric symptoms associated with novel influenza A (H1N1) virus infection. Pediatr Infect Dis J 2010;29:570-571. 